
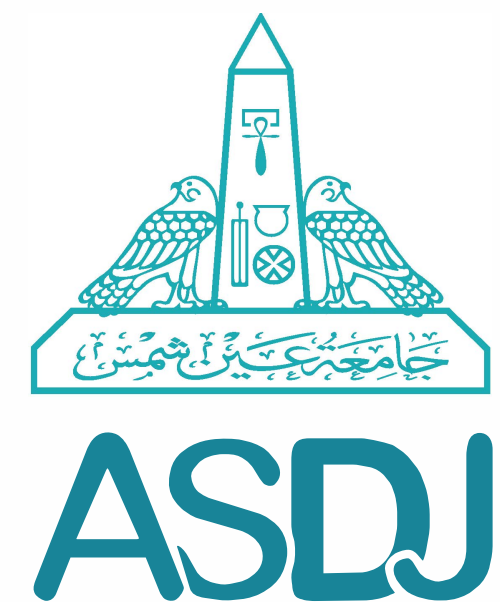

AINSHAMS DENTAL $J O U R$ N A L

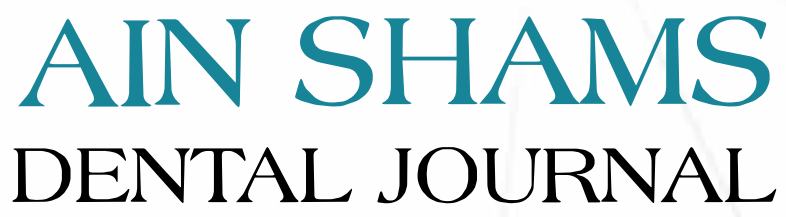

Official Publication of Ain Shams Dental School

December 2020 - Vol. (XXIII) 

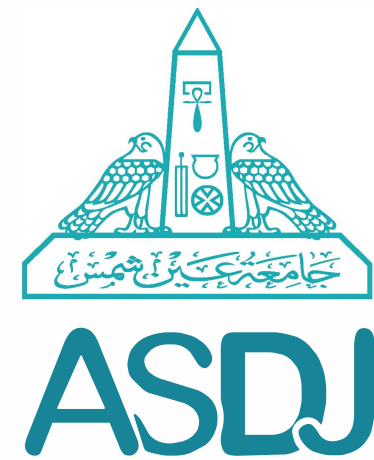

AINSHAMS DENTAL

$J O U R N$ A L

\title{
Executive Board
}

\author{
President \\ Prof. Dr. Mohamed Diaa
}

\author{
Vice President \\ Prof. Dr. Rami Maher Ghali
}

Editor

Dr Ghada Abd El Fattah 


\section{Scientific Board}

\begin{tabular}{|c|c|c|}
\hline \multicolumn{3}{|l|}{ Editor In Chief } \\
\hline Dr.Ghada Abdel Fattah Hussein & ghada.abdelstar@dent.asu.edu.eg & Lecturer of Fixed Prosthodontics \\
\hline \multicolumn{3}{|l|}{ Associate editors } \\
\hline Dr Ahmed osama & ahmed osama@dentasu.edu.eg & Asst.prof. of removable prosthodontics \\
\hline Dr Dina Mohamed Abdel Khalik & dinamohamed@dent.asu.edu.eg & Asst.prof.of Oral Biology \\
\hline Dr Ramy Gaber & amrghanem@dent.asu.edu.eg & Lecturer of Oral \&Maxillofacial Surgery \\
\hline Dr Mohamed Mokhtar Nagui & mohamed.nagy@dent.asu.edu.eg & Asst.prof.of Endodontics \\
\hline Dr Mohamed Kandil & mohamed.kandil@dent.asuedueg & Asst.prof.of dental biomaterials \\
\hline Dr Ahmed Mohsen Foad & ahmed.fovad@dent.asuedueg & Lecturer.of Fixed Prosthodontics \\
\hline Dr Eman Moheb & imanmoheb@dent.asu.edu.eg & Lecturer.of Fixed Prosthodontics \\
\hline Dr Reham Abo El Fadl & rehamabevelfadl@dentasuedueg & Asst.prof. of Pedodontics \\
\hline Dr. Shaimaa Abuelsadat & shaimaa.abuelsadat@dent.asu.edu.eg & Lecturer of Oral Radiology \\
\hline Dr. ZainabMohamed Diaa & zainabdiaa@dent.asu.edu.eg & Lecturer of Operative Dentistry \\
\hline Dr.Ahmed Amro & ahmedamr@dent.asu.edu.eg & Lecturer of Oral medicine and periodontology \\
\hline Dr Noha Ibrahim Abdelrahman & noha.i.abdelrahman@dent.asu.edu.eg & Asst.prof of orhtodontics \\
\hline Dr Marwa Salah & marwasalah@dent.asu.edu.eg & lecturer of Orhtodontics \\
\hline \multicolumn{3}{|l|}{ Editorial Board } \\
\hline \multicolumn{3}{|c|}{ Fixed Prosthodontics, Conservative and Endodontics section } \\
\hline Prof.Dr Tarek Salah EIDin Morsi & tarek.salah@dent.asu.edu.eg & Prof.of Fixed prosthodontics \\
\hline Dr.Maram Mohamed Farouk & maramobeid@dent.asu.edu.eg & Asst.prof.of endodontics \\
\hline Dr. Mohamed Amro & \multirow[t]{2}{*}{ Mohamed.amr@dent.asuedueg } & \multirow[t]{2}{*}{ Lecturer of Operative Dentistry } \\
\hline Prosthodontics section & & \\
\hline Prof.Dr Inji Amin Talaat & \multirow[t]{2}{*}{ ingvtalaat@dent.asuedueg } & \multirow[t]{2}{*}{ Prof.of removable prosthodontics } \\
\hline Oral Medicine, Periodontolgyand Radiology Section & & \\
\hline Prof.Dr.Suzan seif Allah & suzan.seifallah@dent.asu.edu.eg & Prof.of Oral Medicine and Periodontology \\
\hline Dr.Fatma Mostafa Elbadawy & fatmaelbadawy@dent.asu.edu.eg & Lecturer of Oral Radiology \\
\hline Pedodontics and Orthodontics Sectic & & \\
\hline Prof.Dr.Noha Sabet & nohasabet@dent.asu.edu.eg & Prof.of Orthodontics \\
\hline Dr.AmiraSaad Ahmed Badran & dr.amirasaad@dent.asu.edu.eg & Asst.prof.of Pedodontics \\
\hline Oral and Maxillofacial Surgery Sectic & & \\
\hline Prof.Dr.Marwa Elkassaby & marwaelkassaby@dent.asu.edu.eg & Prof.of Oral And Maxillofacial surgery \\
\hline Dental Biomaterial Science Section & & \\
\hline Prof.Dr.Mohamed Salah Abdel aziz & mohamednassif@dent.asuedueg & Prof.of Dental biomaterials \\
\hline Cellular Histopathology Section & & \\
\hline Dr.Marwa Mohamed Abd elHamid & mamamiro@dent.asuedueg & Asst.prof.of Oral biology \\
\hline Dr. Basma Abdel Rhman Ahmed & basmaabdelrahman@dent.asu.edu.eg & Lecturer of Oral pathology \\
\hline Technical Editors & & \\
\hline Mr.Mohamed Fawzy & SupportASDJ@dent.asu.eg & \\
\hline Mr. Khaled Ragab & Accounting.ASDJ@dent.asu.edu.eg & \\
\hline Miss Hilana safwathelmy & Info_ASDJ@dent.asu.edu.eg & \\
\hline
\end{tabular}

